\title{
Baicalein induces apoptosis via a mitochondrial-dependent caspase activation pathway in T24 bladder cancer cells
}

\author{
HONG-LIANG LI ${ }^{1,2}$, SHU ZHANG ${ }^{2,3}$, YUAN WANG ${ }^{4}$, RONG-RUI LIANG ${ }^{2,3}$, JUN LI ${ }^{2}$, \\ PENG AN ${ }^{2}$, ZI-MING WANG ${ }^{1}$, JUN YANG ${ }^{2,5}$ and ZONG-FANG LI ${ }^{2,3}$

\begin{abstract}
${ }^{1}$ Department of Urology, Second Affiliated Hospital, College of Medicine, Xi'an Jiaotong University, Xi'an 710004;
${ }^{2}$ Engineering Research Center of Biotherapy and Translational Medicine of Shaanxi Province, Xi'an Jiaotong University, Xi'an 710004; ${ }^{4}$ Department of Pathology, Shaanxi Provincial People's Hospital, Xi'an 710068; ${ }^{5}$ Department of Pathology, Second Affiliated Hospital, College of Medicine, Xi'an Jiaotong University, Xi'an 710004, P.R. China
\end{abstract} \\ Xi'an 710004; ${ }^{3}$ Department of General Surgery, Second Affiliated Hospital, College of Medicine, Xi'an Jiaotong University,
}

Received July 3, 2012; Accepted September 20, 2012

DOI: $10.3892 / \mathrm{mmr} .2012 .1123$

\begin{abstract}
Recurrence of bladder cancer following transurethral resection of bladder tumor (TURBt) is an obstacle in clinical management. In the current study, we investigated the antitumor activity of baicalein, a Chinese herbal medicine, against T24 bladder cancer cells in vitro. Baicalein inhibited growth and caused G1/S arrest of the cell cycle in the T24 cells. Moreover, baicalein induced apoptosis via loss of mitochondrial transmembrane potential $(\Delta \Psi \mathrm{m})$, release of cytochrome $\mathrm{c}$ and activation of caspase- 9 and caspase-3. Baicalein inhibited Akt phosphorylation, downregulated $\mathrm{Bcl}-2$ expression and upregulated Bax expression, which in turn increased the ratio of Bax/Bcl-2. Our results demonstrate that baicalein repressed growth inhibition and induced apoptosis via loss of $\Delta \Psi \mathrm{m}$ and activation of caspase- 9 and caspase- 3 in T24 bladder cancer cells, which indicates that baicalein may be an effective agent in the clinical management of bladder cancer.
\end{abstract}

\section{Introduction}

Bladder cancer is a common malignancy of the urologic system. Approximately $75 \%$ of new patients have non-muscle-invasive bladder cancer, and transurethral resection of bladder tumor (TURBt) is the standard treatment for those patients (1). However, following surgery, $60-70 \%$ of the cancers will recur, with $25 \%$ exhibiting a higher stage or grade, which remains a problem for surgeons (2). Therefore,

Correspondence to: Professor Zong-Fang Li, Engineering Research Center of Biotherapy and Translational Medicine of Shaanxi Province; Department of General Surgery, Second Affiliated Hospital, School of Medicine, Xi'an Jiaotong University, 157 West Fifth Road, Shaanxi, Xi'an 710004, P.R. China

E-mail:1zf2568@gmail.com

Key words: baicalein, bladder cancer, Akt, Bcl-2, apoptosis intravesical chemotherapy is widely used as an adjuvant treatment to prevent recurrence and cancer progression following TURBt (3). However, the cytotoxicity and incomplete efficacy of chemical agents limits their use as regular intravesical drugs, even if they induce apoptosis of the cancer cells (3). Thus, new intravesical agents are urgently required for the clinical management of bladder cancer.

Baicalein is a flavonoid derived from the root of Scutellaria baicalensis, a plant widely used in Chinese herbal medicine. Baicalein is a well-known inhibitor of 12-lipoxygenase (12-LOX) (4), the expression of which correlates with tumorigenicity and tumor progression $(5,6)$. Previous studies have shown that baicalein exerts numerous biological activities, including anti-inflammatory, anti-viral and antioxidant activities (7-9). Furthermore, other studies have indicated that this flavonoid also inhibits cancer cell growth and induces apoptosis in human breast, prostate, hepatocellular and myeloma cancer cells (10-13). However, its antitumor role in bladder cancer is still unclear.

In the current study, we report that baicalein suppresses the growth and clone-forming ability of T24 bladder cancer cells in vitro. Additionally, T24 cells are arrested at the G1/S phase and undergo apoptosis following baicalain treatment. Baicalein inhibits Akt phosphorylation, activates caspase-9 and caspase- 3 and downregulates Bcl-2 expression.

\section{Materials and methods}

Cell culture and reagents. T24 human bladder cancer cells were obtained from American Type Culture Collection (Manassas, VA, USA) and maintained in DMEM (Gibco, Grand Island, NY, USA) supplemented with $10 \%$ fetal bovine serum (FBS; Sijiqing, Hangzhou, China) at $3{ }^{\circ} \mathrm{C}$ with $5 \% \mathrm{CO}_{2}$ in a humidified incubator. Baicalein (purity $>98 \%$ ) and mytomycin were purchased from Sigma-Aldrich (St. Louis, MO, USA) and dissolved in dimethyl sulfoxide (DMSO). Primary antibodies for Akt, phosphorylated Akt, Bcl-2 and Bax were purchased from Santa Cruz Biotechnology, Inc. (Santa Cruz, CA, USA). The study was approved by the Ethical Review 
A

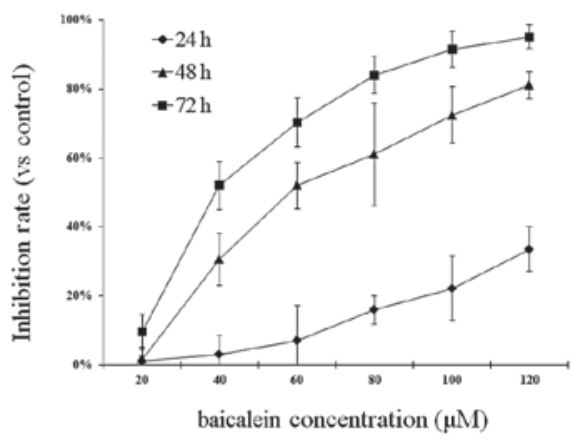

B

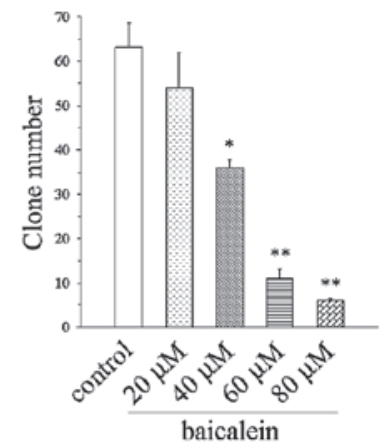

Figure 1. Baicalein decreased the growth of T24 bladder cancer cells in vitro. (A) T24 cells were treated with various concentrations of baicalein and the cell inhibition rate was determined by MTT assay. (B) The colony-forming ability in response to baicalein treatment was determined as detailed in Materials and methods. ${ }^{*} \mathrm{P}<0.05,{ }^{* *} \mathrm{P}<0.01$, compared with the control cells.

Board (ERB) committee (The Second Affiliated Hospital of Medical College, Xi'an Jiaotong University, Xi'an, China)

Cell viability assay. The T24 cells were seeded in 96-well plates at a density of $1 \times 10^{4}$ cells per well and exposed to $0-120 \mu \mathrm{mol} / 1$ baicalein for $24 \mathrm{~h}$. The cells were then washed with PBS twice and recultured for 3 days. MTT (final concentration $0.5 \mathrm{mg} / \mathrm{ml}$; Sigma-Aldrich) was then added, the cells were incubated for a further $4 \mathrm{~h}$, and finally the formazan crystals was dissolved in DMSO. The optical density (OD) value was measured at $490 \mathrm{~nm}$ using a microplate autoreader (BioTek Instruments, Inc., Winooski, VT, USA) and the relative cell viability was calculated as a percentage. Independent experiments were repeated in triplicate.

Colony formation assay. After pre-treatment with baicalein (0-80 $\mu \mathrm{mol} / \mathrm{l})$ for $24 \mathrm{~h}, 1,000$ cells per well of a T24 single-cell suspension were seeded in 6 -well plates and incubated at $37^{\circ} \mathrm{C}$ with $5 \% \mathrm{CO}_{2}$ in a humidified incubator for 14 days; fresh medium was added every 4 days. The plates were then washed with icecold PBS, fixed with $4 \%$ paraformaldehyde, stained with crystal violet solution for $15 \mathrm{~min}$ at room temperature and washed with distilled water until no color was evident in the rinse. Plates were dried in air and the colony numbers were counted.

Cell cycle assay. T24 bladder cancer cells were plated in $60-\mathrm{mm}$ dishes and treated with $0-80 \mu \mathrm{mol} / \mathrm{l}$ baicalein for $24 \mathrm{~h}$. The cells were then harvested, fixed with ice-cold $70 \%$ ethanol and incubated with $4 \mu \mathrm{g} / \mathrm{ml}$ propidium iodide (PI) solution in the presence of $100 \mu \mathrm{g} / \mathrm{ml}$ RNase for $30 \mathrm{~min}$. The samples were analyzed using a flow cytometer (FACScan; Becton-Dickinson, San Jose, CA, USA). Three independent experiments were performed.

Apoptosis and mitochondrial transmembrane potential $(\Delta \Psi \mathrm{m})$ assay. Following treatment with $0-80 \mu \mathrm{mol} / \mathrm{l}$ baicalein for $24 \mathrm{~h}$, the T2 4 cells were harvested and washed with PBS. An apoptosis detection kit (Invitrogen, Carlsbad, CA, USA) containing annexin V-FITC and PI was used to identify apoptotic cells according to the manufacturer's instructions. The $\Delta \Psi \mathrm{m}$ was determined by JC-1 staining. Data were collected by flow cytometric analysis using a FACSCalibur flow cytometer (Becton-Dickinson). For each assay, independent experiments were repeated in triplicate.
Caspase-9 and -3 activity assay. T24 cells were incubated with $0-80 \mu \mathrm{mol} / 1$ baicalein for $24 \mathrm{~h}$ and cellular extracts were then obtained. Caspase- 9 and -3 activities were determined using a human caspase- 9 and -3 (active) ELISA kit (Invitrogen) according to the manufacturer's instructions. The OD value was then measured at $450 \mathrm{~nm}$ using a Microplate Autoreader. Three independent experiments were performed.

Western blot assay. T24 cells were incubated with $0-80 \mu \mathrm{mol} / 1$ baicalein for $24 \mathrm{~h}$. Total cellular extracts were then prepared using RIPA buffer containing proteinase inhibitors. Cytosol/mitochondria fractionation was carried out using a mitochondria extraction kit (Runtai Biotech, Tianjin, China). Equal amounts of lysates $(30 \mu \mathrm{g})$ were separated by $12 \%$ SDS-PAGE and transferred to nitrocellulose membranes. The membranes were initially blocked with $5 \%$ skimmed milk in TBS for $1 \mathrm{~h}$ at room temperature and then incubated with primary antibodies at $4^{\circ} \mathrm{C}$ overnight followed by secondary antibodies coupled with horseradish peroxidase for $1 \mathrm{~h}$ at room temperature. Protein signals were then detected using an ECL chemiluminescent detection system (Amersham, Piscataway, NJ, USA). GAPDH was used as a loading control.

Statistical analysis. All data analyses were performed using SPSS 13.0 software for Windows. $\mathrm{P}<0.05$ was considered to indicate a statistically significant result.

\section{Results}

Baicalein inhibits T24 bladder cancer cell growth capability in vitro. We investigated the effect of baicalein on T24 human bladder cancer cell growth. As shown in Fig. 1A, the rate of cell growth was reduced by treatment with baicalein in a concentration- and time-dependent manner. High concentrations (80-120 $\mu \mathrm{mol} / \mathrm{l})$ almost completely blocked cell proliferation. Colony formation following baicalein treatment was also determined. The results revealed that baicalein repressed the clonogenicity of the T24 cells in a concentration-dependent manner (Fig. 1B).

Baicalein increases G1/S arrest and induces apoptosis. Since baicalein was able to suppress the proliferation of the T24 cells, we next investigated its effect on the cell cycle. As shown in Fig. 2A, the T24 cells were arrested at the G1/S 


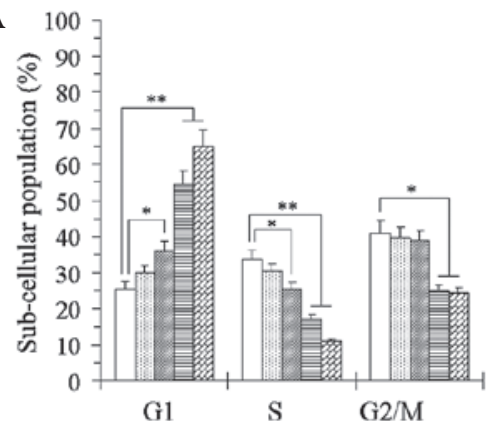

B

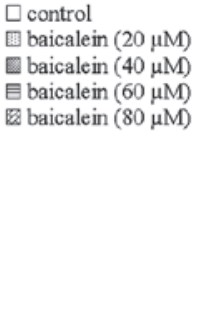

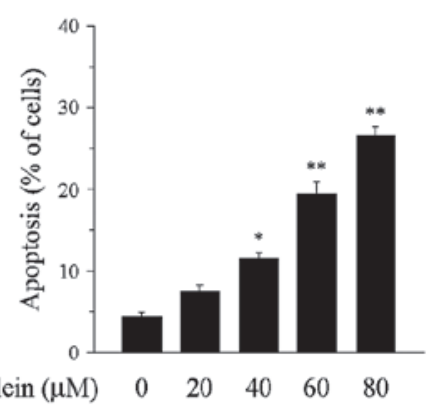

Figure 2. Baicalein induced cell cycle arrest and apoptosis in T24 bladder cancer cells. Following treatment with $0-80 \mu$ mol/1 baicalein for $24 \mathrm{~h}$ the cells were stained with (A) propidium iodide (PI) or (B) annexin V-FITC/PI and analyzed by flow cytometry. ${ }^{*} \mathrm{P}<0.05,{ }^{* *} \mathrm{P}<0.01$, compared with the control cells.

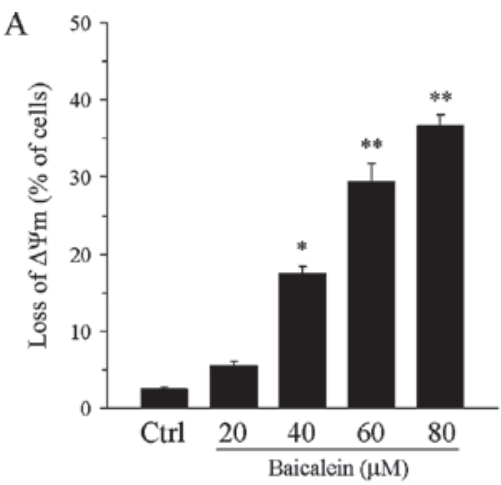

$\mathrm{C}$

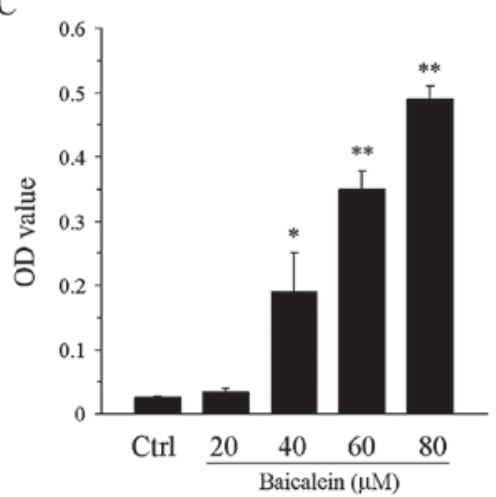

B

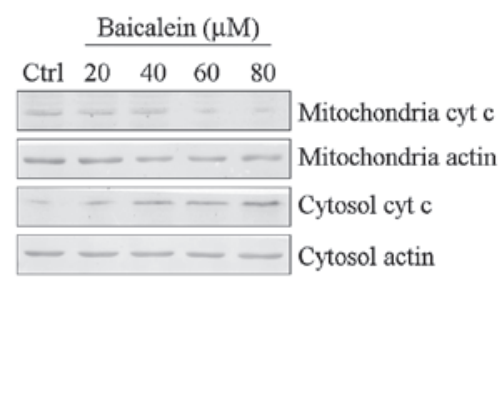

$\mathrm{D}$

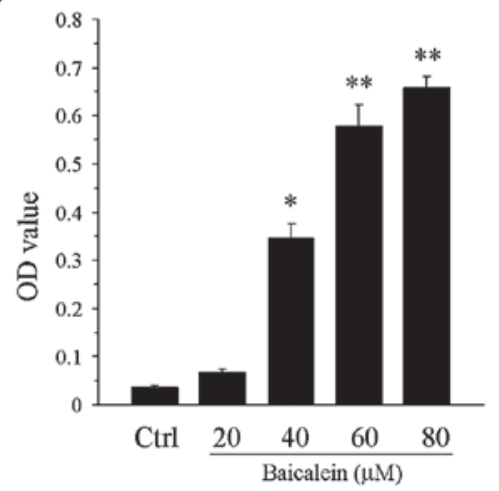

Figure 3. Baicalein induces mitochondrial transmembrane potential $(\Delta \Psi \mathrm{m})$ collapse and activates caspase- 9 and -3 . (A) Following treatment with $0-80 \mu$ mol/1 baicalein for $24 \mathrm{~h}, \mathrm{~T} 24$ cells were harvested, stained with JC-1 and analyzed by flow cytometry. (B) Cytosol/mitochondria fractionation was performed as detailed in Materials and methods. Release of cytochrome $\mathrm{c}$ was determined by western blot analysis. (C and D) The activities of caspase-9 and -3 were detected by ELISA. ${ }^{*} \mathrm{P}<0.05,{ }^{* *} \mathrm{P}<0.01$, compared with the control cells.

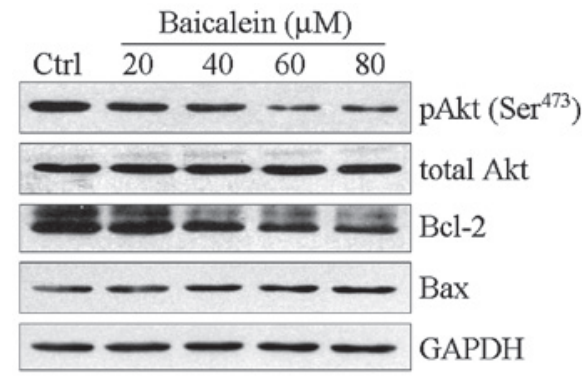

Figure 4. Baicalein inhibits Akt phosphorylation and downregulates Bcl-2 expression. T24 cells were incubated with $0-80 \mu \mathrm{mol} / 1$ baicalein for $24 \mathrm{~h}$ and total cellular extracts were obtained. Phosphorylated Akt $\left(\operatorname{Ser}^{473}\right)$, total Akt, Bcl-2 and Bax were determined by western blot analysis. phase following baicalein treatment. In addition, the T24 cells underwent cellular apoptosis following baicalein treatment. The levels of apoptosis were significantly higher in the cells treated with 60 or $80 \mu \mathrm{mol} / 1$ baicalein than in the control cells $(\mathrm{P}<0.05$ and $\mathrm{P}<0.01$, respectively; Fig. $2 \mathrm{~B})$.

Baicalein induces $\Delta \Psi m$ collapse and activates caspase- 9 and -3 . Since the loss of $\Delta \Psi \mathrm{m}$ plays a critical role in triggering apoptosis, we next examined the effect of baicalein treatment on $\Delta \Psi \mathrm{m}$. Following treatment with baicalein for $24 \mathrm{~h}$, a significant concentration-dependent loss of $\Delta \Psi \mathrm{m}$ was observed in the treated cells compared with the control cells $(\mathrm{P}<0.05$ or $\mathrm{P}<0.01$; Fig. 3A). The release of cytochrome $\mathrm{c}$ from the mitochondria to the cytoplasm was also observed (Fig. 3B). 
Furthermore, we determined the activities of caspase- 9 and -3 following baicalein treatment. Consistent with the $\Delta \Psi$ m result, the baicalein-treated T24 cells exhibited elevated caspase- 9 and -3 activities compared with the control cells $(\mathrm{P}<0.05$ or $\mathrm{P}<0.01$; Fig. 3C and D).

Baicalein inhibits Akt phosphorylation and downregulates Bcl-2 expression. Since previous reports have indicated that the PI3K/Akt pathway provides a survival signal to protect cells from apoptosis (14), we determined the effect of baicalein treatment on the activity of Akt. As shown in Fig. 4, baicalein repressed Akt $\left(\mathrm{Ser}^{473}\right)$ phosphorylation in a concentration-dependent manner. Bcl-2 and Bax belong to the $\mathrm{Bcl}-2$ family. Bcl-2 is an anti-apoptotic protein whereas Bax is a pro-apoptotic protein. The $\mathrm{Bax} / \mathrm{Bcl}-2$ ratio is significant in apoptosis $(15,16)$. Therefore, we examined the alterations in the levels of these proteins in response to baicalein exposure. The expression levels of Bcl-2 were significantly decreased following baicalein treatment, whereas those of Bax were upregulated.

\section{Discussion}

The high recurrence of bladder cancer following TURBt is an obstacle in its clinical treatment. Intravesical chemotherapy is used to prevent recurrence; however, tumor cells often re-emerge, even in those patients undergoing regular intravesical chemotherapy (3). Therefore, an effective therapeutic strategy is urgently required. In the current study, we report the ability of a Chinese herbal medicine, baicalein, to inhibit growth and induce apoptosis in T24 bladder cancer cells, which indicates its potential as an antitumor agent for the treatment of bladder cancer.

In an MTT assay, after incubating T24 cells with baicalein, the tumor cells displayed a decreased growth rate compared with the control cells. Moreover, high concentrations of baicalein almost completely blocked cell growth. These data indicate that baicalein is able to inhibit the growth of T24 bladder cancer cells. In addition, clonogenicity was also suppressed following baicalein treatment. These data suggest that baicalein is able to retard the growth of T24 cells in vitro.

Tumor progression is dependent on the balance between proliferating and apoptotic cells (17). Chemotherapy may induce cell cycle arrest and cellular apoptosis in tumor cells, thereby altering the ratio of proliferating to apoptotic cells and leading to repression of the tumor. In the present study, T24 bladder cancer cells arrested at the G1/S phase following incubation with baicalein for $24 \mathrm{~h}$. Furthermore, the T24 cells underwent apoptosis in response to baicalein treatment. Notably, baicalein induces cell cycle arrest and apoptosis in a concentration-dependent manner. Previous reports have shown that $\Delta \Psi$ m plays an initial role in the apoptotic cascade (18). In this study, we investigated the effect of baicalein on $\Delta \Psi \mathrm{m}$. As we expected, the T24 cells exhibited a significant $\Delta \Psi \mathrm{m}$ collapse following baicalein treatment. Correspondingly, elevated activities of caspase- 9 and -3 were observed in the T24 cells following incubation with baicalein. Since activation of caspase-9 is a marker of intrinsic apoptosis (19), we propose that baicalein is capable of inducing intrinsic apoptosis in T24 bladder cancer cells.
Consistent activation of the PI3K/Akt pathway has been detected in numerous human tumor cells, including bladder cancer $(20,21)$. This signal pathway promotes tumor survival, progression and metastasis $(22,23)$. Akt regulates several cellular activities, including proliferation, the cell cycle and apoptosis $(22,23)$. In the current study, we demonstrated that baicalein repressed Akt phosphorylation in a concentrationand time-dependent manner. The Bcl-2 family consists of several proteins that execute pro- and anti-apoptotic functions (24,25). Bcl-2 is a member of this family and serves as an anti-apoptotic protein (25). Previous studies have indicated that $\mathrm{Bcl}-2$ plays a critical role in the survival, anti-apoptotic activity and chemoresistance of tumor cells $(26,27)$. Another $\mathrm{Bcl}-2$ family member, Bax, is a pro-apoptotic protein, which forms a heterodimer with $\mathrm{Bcl}-2$ and thus represses its antiapoptotic function $(28,29)$. Therefore, the $\mathrm{Bcl}-2 / \mathrm{Bax}$ ratio is a key factor in apoptosis. In the present study, we found that Bcl-2 expression was suppressed whereas that of Bax was slightly upregulated following baicalein treatment. Based on these results, we propose that baicalein induces cell cycle arrest and apoptosis through the inhibition of Akt and Bcl-2 in T24 bladder cancer cells.

In conclusion, we have shown that baicalein leads to growth inhibition due to cell cycle arrest and apoptosis via the loss of $\Delta \Psi \mathrm{m}$ and activation of caspase-9 and -3 in T24 bladder cancer cells. Moreover, baicalein treatment is able to inhibit Akt phosphorylation and downregulate $\mathrm{Bcl}-2$ expression. These results indicate that baicalein may be an effective agent in the clinical management of bladder cancer and expand our understanding of the potential clinical applications of baicalein.

\section{Acknowledgements}

This study received financial support from the Program for Changjiang Scholars and Innovative Research Team in University (PCSIRT:1171) and Shaanxi Province Science and Technology Research and Development Program (No. 2009K12-01).

\section{References}

1. Bischoff CJ and Clark PE: Bladder cancer. Curr Opin Oncol 21: 272-277, 2009.

2. Schenk-Braat EA and Bangma CH: Immunotherapy for superficial bladder cancer. Cancer Immunol Immunother 54: 414-423, 2005.

3. Shen Z, Shen T, Wientjes MG, O'Donnell MA and Au JL: Intravesical treatments of bladder cancer: review. Pharm Res 25: 1500-1510, 2008

4. Sekiya K and Okuda H: Selective inhibition of platelet lipoxygenase by baicalein. Biochem Biophys Res Commun 105: 1090-1095, 1982.

5. Pidgeon GP, Kandouz M, Meram A and Honn KV: Mechanisms controlling cell cycle arrest and induction of apoptosis after 12-lipoxygenase inhibition in prostate cancer cells. Cancer Res 62: 2721-2727, 2002.

6. Wong BC, Wang WP, Cho CH, Fan XM, Lin MC, Kung HF and Lam SK: 12-Lipoxygenase inhibition induced apoptosis in human gastric cancer cells. Carcinogenesis 22: 1349-1354, 2001.

7. Chen SF, Hsu CW, Huang WH and Wang JY: Post-injury baicalein improves histological and functional outcomes and reduces inflammatory cytokines after experimental traumatic brain injury. Br J Pharmacol 155: 1279-1296, 2008.

8. Boyle SP, Doolan PJ, Andrews CE and Reid RG: Evaluation of quality control strategies in Scutellaria herbal medicines. J Pharm Biomed Anal 54: 951-957, 2011. 
9. He XL, Wang YH, Gao M, Li XX, Zhang TT and Du GH: Baicalein protects rat brain mitochondria against chronic cerebral hypoperfusion-induced oxidative damage. Brain Res 1249: 212-221, 2009.

10. Wang L, Ling Y, Chen Y, et al: Flavonoid baicalein suppresses adhesion, migration and invasion of MDA-MB-231 human breast cancer cells. Cancer Lett 297: 42-48, 2010.

11. Taniguchi H, Yoshida T, Horinaka M, et al: Baicalein overcomes tumor necrosis factor-related apoptosis-inducing ligand resistance via two different cell-specific pathways in cancer cells but not in normal cells. Cancer Res 68: 8918-8927, 2008.

12. Chen CH, Huang LL, Huang CC, Lin CC, Lee Y and Lu FJ: Baicalein, a novel apoptotic agent for hepatoma cell lines: a potential medicine for hepatoma. Nutr Cancer 38: 287-295, 2000

13. Ma Z, Otsuyama K, Liu S, et al: Baicalein, a component of Scutellaria radix from Huang-Lian-Jie-Du-Tang (HLJDT), leads to suppression of proliferation and induction of apoptosis in human myeloma cells. Blood 105: 3312-3318, 2005.

14. Carnero A: The PKB/AKT pathway in cancer. Curr Pharm Des 16: 34-44, 2010.

15. Czabotar PE and Lessene G: Bcl-2 family proteins as therapeutic targets. Curr Pharm Des 16: 3132-3148, 2010.

16. Kirkin V, Joos S and Zörnig M: The role of Bcl-2 family members in tumorigenesis. Biochim Biophys Acta 1644: 229-249, 2004.

17. Malaguarnera L: Implications of apoptosis regulators in tumorigenesis. Cancer Metastasis Rev 23: 367-387, 2004.

18. Tsujimoto Y and Shimizu S: Role of the mitochondrial membrane permeability transition in cell death. Apoptosis 12: 835-840, 2007.

19. Hengartner MO: The biochemistry of apoptosis. Nature 407: 770-776, 2000

20. Goswami A, Ranganathan P and Rangnekar VM: The phosphoinositide 3-kinase/Akt1/Par-4 axis: a cancer-selective therapeutic target. Cancer Res 66: 2889-2892, 2006.
21. Chen M, Gu J, Delclos GL, Killary AM, et al: Genetic variations of the PI3K-AKT-mTOR pathway and clinical outcome in muscle invasive and metastatic bladder cancer patients. Carcinogenesis 31: 1387-1391, 2010

22. Jiang BH and Liu LZ: PI3K/PTEN signaling in angiogenesis and tumorigenesis. Adv Cancer Res 102: 19-65, 2009.

23. Hartmann W, Küchler J, Koch A, et al: Activation of phosphatidylinositol-3'-kinase/AKT signaling is essential in hepatoblastoma survival. Clin Cancer Res 15: 4538-4545, 2009.

24. Danial NN: BCL-2 family proteins: critical checkpoints of apoptotic cell death. Clin Cancer Res 13: 7254-7263, 2007.

25. Yang J, Liu X, Bhalla K, et al: Prevention of apoptosis by Bcl-2: Release of cytochrome c from mitochondria blocked. Science 275: 1129-1132, 1997.

26. Skommer J, Brittain T and Raychaudhuri S: Bcl-2 inhibits apoptosis by increasing the time-to-death and intrinsic cell-to-cell variations in the mitochondrial pathway of cell death. Apoptosis 15: 1223-1233, 2010.

27. Zhao Y, Zhang CL, Zeng BF, Wu XS, Gao TT and Oda Y: Enhanced chemosensitivity of drug-resistant osteosarcoma cells by lentivirus-mediated Bcl-2 silencing. Biochem Biophys Res Commun 390: 642-647, 2009

28. Reagan-Shaw S, Nihal M, Ahsan H, Mukhtar H and Ahmad N: Combination of vitamin $\mathrm{E}$ and selenium causes an induction of apoptosis of human prostate cancer cells by enhancing $\mathrm{Bax} / \mathrm{Bcl}-2$ ratio. Prostate 68: 1624-1634, 2008.

29. Katiyar SK, Roy AM and Baliga MS: Silymarin induces apoptosis primarily through a p53-dependent pathway involving $\mathrm{Bcl}-2 / \mathrm{Bax}$, cytochrome $\mathrm{c}$ release, and caspase activation. Mol Cancer Ther 4: 207-216, 2005. 